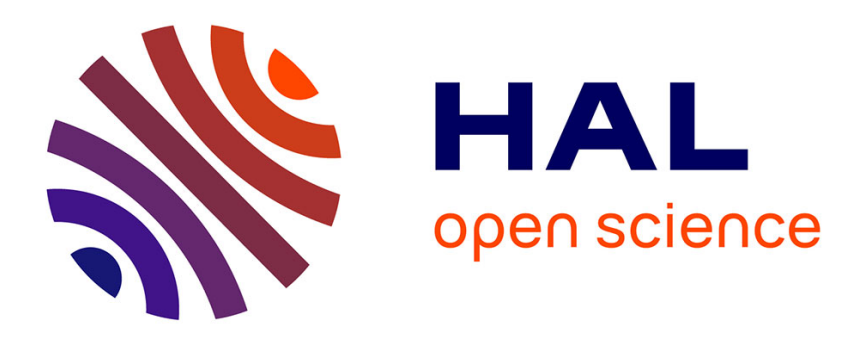

\title{
Impact de deux orthèses d'avancée mandibulaire sur l'articulation temporomandibulaire
}

\author{
L. Cheze, B. Navailles
}

\section{To cite this version:}

L. Cheze, B. Navailles. Impact de deux orthèses d'avancée mandibulaire sur l'articulation temporomandibulaire. Innovation et Technologie en Biologie et Médecine;ITBM-RBM, 2006, Vol27, issue 5-6, p233-7. hal-00505756

\section{HAL Id: hal-00505756 https://hal.science/hal-00505756}

Submitted on 26 Jul 2010

HAL is a multi-disciplinary open access archive for the deposit and dissemination of scientific research documents, whether they are published or not. The documents may come from teaching and research institutions in France or abroad, or from public or private research centers.
L'archive ouverte pluridisciplinaire HAL, est destinée au dépôt et à la diffusion de documents scientifiques de niveau recherche, publiés ou non, émanant des établissements d'enseignement et de recherche français ou étrangers, des laboratoires publics ou privés. 
ITBM-RBM, Vol. 27, Issues 5-6, Nov-Dec- 2006, P- 233-237 doi:10.1016/j.rbmret.2007.01.004

Copyright $\odot 2007$ Elsevier Masson SAS All rights reserved.

Elsevier Editorial System(tm) for ITBM-RBM, Innovation et Technologie en Biologie et Médecine

Manuscript Draft

Manuscript Number: RBMRET-D-07-00001R1

Title: Impact de deux orthèses d'avancée mandibulaire sur l'articulation temporo-mandibulaire

Article Type: Article original

Section/Category:

Keywords: Apnées obstructives du sommeil, Avancée mandibulaire, Orthèse endo-buccale, Forces musculaires, ATM.

Corresponding Author: Professeur Laurence CHEZE, Ph.D.

Corresponding Author's Institution: Université Lyon 1

First Author: Laurence CHEZE, Professeur

Order of Authors: Laurence CHEZE, Professeur; Bruno NAVAILLES, MD

Manuscript Region of Origin:

Abstract: Objectif : Analyser les efforts appliqués par deux types d'orthèses d'avancée mandibulaire sur l'articulation temporo-mandibulaire.

Matériel \& Méthodes : Un modèle mécanique plan de l'articulation temporo-mandibulaire, de type corps rigides, prenant en compte six muscles, est développé. Une comparaison des efforts musculaires et articulaires, en position de protrusion de $10 \mathrm{~mm}$, est réalisée entre deux types d'orthèses : l'une travaillant en poussée et l'autre en retenue. Dans les deux cas, l'équilibre statique conduit à un système d'équations hyperstatique qui est résolu par optimisation numérique sous contraintes, en minimisant différents critères. 
Résultats : Pour l'orthèse travaillant en poussée, les résultats montrent qu'un effort important est appliqué par le masseter et le posterior temporal. Ces deux muscles étant élévateurs de la mandibule, ceci implique une ouverture de la bouche avec ce type d'orthèse lorsque les muscles sont au repos. Au contraire, l'orthèse travaillant en retenue permet d'obtenir une protrusion de $10 \mathrm{~mm}$ avec un effort minimal dans ces muscles. De plus, l'action de contact sur l'articulation temporo-mandibulaire est en moyenne plus faible de $10 \%$ avec l'orthèse travaillant en retenue.

Conclusion : Le modèle mécanique simple proposé permet la comparaison d'orthèses ayant des modes d'action différents, et les résultats trouvés sont en accord avec ceux de la littérature. Les résultats sur les paramètres étudiés (ouverture de la bouche, action de contact articulaire) tendent à montrer un meilleur profil clinique en termes de compliance et d'effets secondaires des orthèses travaillant en retenue par rapport à celles travaillant en compression. 
Titre :

Impact de deux orthèses d'avancée mandibulaire sur l'articulation temporomandibulaire

Mots-clés :

Apnées Obstructives du Sommeil, Avancée mandibulaire, Orthèse endo-buccale, Forces musculaires, ATM.

\section{Auteurs :}

Laurence CHEZE, PhD

Laboratoire de Biomécanique et Mécanique des Chocs

Université Claude Bernard - Lyon 1 / INRETS, Villeurbanne

Bruno Navailles, MD

Service ORL, Centre hospitalier de Valence

\section{$\underline{\text { Correspondance à adresser à : }}$}

Pr. Laurence CHEZE

Laborato ire de Biomécanique et Mécanique des Chocs

Bât. Omega, Université Claude Bernard - Lyon 1

43 Bd. du 11 novembre 1918

69622 Villeurbanne Cedex

Tel : 0472448098

Fax : 0472448054

Email : Laurence.Cheze@univ-lyon1.fr 


\title{
Impact de deux orthèses d'avancée mandibula ire sur l'articulation temporo- mandibulaire
}

\author{
Laurence Chèze ${ }^{a}$, Bruno Navailles ${ }^{b}$ \\ ${ }^{a}$ Université de Lyon, Lyon, F-69000 ; Université Lyon 1, Laboratoire de Biomécanique et \\ Mécanique des Chocs (UCBL / INRETS) \\ ${ }^{b}$ Service ORL, Centre hospitalier de Valence
}

\section{Résumé :}

Objectif : Analyser les efforts appliqués par deux types d'orthèses d'avancée mandibulaire sur l'articulation temporo-mandibulaire.

Matériel \& Méthodes : Un modèle mécanique plan de l'articulation temporo-mandibulaire, de type corps rigides, prenant en compte six muscles, est développé. Une comparaison des efforts musculaires et articulaires, en position de protrusion de $10 \mathrm{~mm}$, est réalisée entre deux types d'orthèses : l'une travaillant en poussée et l'autre en retenue. Dans les deux cas, l'équilibre statique conduit à un système d'équations hyperstatique qui est résolu par optimisation numérique sous contraintes, en minimisant différents critères.

Résultats : Pour l'orthèse travaillant en poussée, les résultats montrent qu'un effort important est appliqué par le masseter et le posterior temporal. Ces deux muscles étant élévateurs de la mandibule, ceci implique une ouverture de la bouche avec ce type d'orthèse lorsque les muscles sont au repos. Au contraire, l'orthèse travaillant en retenue permet d'obtenir une protrusion de $10 \mathrm{~mm}$ avec un effort minimal dans ces muscles. De plus, l'action de contact sur l'articulation temporo-mandibulaire est en moyenne plus faible de $10 \%$ avec l'orthèse travaillant en retenue.

Conclusion : Le modèle mécanique simple proposé permet la comparaison d'orthèses ayant des modes d'action différents, et les résultats trouvés sont en accord avec ceux de la littérature. Les résultats sur les paramètres étudiés (ouverture de la bouche, action de con tact articulaire) tendent à montrer un meilleur profil clinique en termes de compliance et d'effets secondaires des orthèses travaillant en retenue par rapport à celles travaillant en compression.

Mots-clés : Apnées Obstructives du Sommeil, Avancée mandibulaire, Orthèse endo-buccale, Forces musculaires, ATM.

\section{Abstract :}

Objectives: Understand mechanical forces applied on the temporo-mandibular joint by two different design s of mandibular advancement devices.

Methods \& Measurements: Rigid elements model of the temporo-mandibular jo int, taking into account six muscles, was developed. A study is designed to compare traction based vs. compression based devices, with mandible in a $10 \mathrm{~mm}$ protrusion position. 
Static equilibrium can be written as hyperstatic equations and resolution is obtained through numeric optimization of different criteria under constraints.

Results: For compression based device, equation results show that important strength is applied in the masseter and posterior temporal. As both muscles lift the mandible up, this implies mouth opening happens when these muscles are at rest. However, the traction based device enables $10 \mathrm{~mm}$ protrusion with minimal effort on these muscles. Additionally, joint contact strength is consistently less $(10 \%)$ with traction based device than with compression based device.

Conclusions: This simple mechanical model enables comparison of mandibular advancement devices with different modes of action. The results found are consistent with the ones from literature. Findings on studied parameters (mouth opening, jo int contact strength) may imply an improved side-effect and a compliance profile in clinical practice for traction-based over compression-based mechan isms.

Key-words : Obstructive Sleep Apnea, Mandibular Advancement, Mandibular Repositioning Appliance, Muscular Strength, TMJ.

\section{$\underline{\text { Introduction }}$}

Le syndrome d'apnées obstructives du sommeil est une pathologie qui touche 2 à $4 \%$ de la population adulte [1] et dont les conséquences sur les troubles du sommeil et le système cardio-vasculaires sont démontrées. Le traitement de référence est la ventilation par pression positive continue (VPPC), obtenue par une attelle pneumatique prévenant le collapsus pharyngé. Le traitement alternatif est l'orthèse d'avancée mandibulaire (OAM) - attelle mécanique qui diminue le risque de collapsus pharyngé en augmentant le calibre des voies aériennes supérieures. Depuis les premiers travaux de Meier-Ewert en 1984, de nombreuses études ont montré l'efficacité des OAM dans le syndrome d'apnées obstructives du sommeil ainsi qu'une préférence des patients pour l'orthèse par rapport à la VPPC [2]. Les effets secondaires à court terme (douleurs dentaires, sécheresse en bouche, salivation excessive) et à long-terme (modification de l'occlusion, inclinaison des incisives) ont été également largement étudiés et varient suivant les études et les orthèses étudiées [3]. L'observance varie en tre 55 et $82 \%$ après un an de traitement [4].

Néanmoins il existe très peu de travaux et d'études comparatives entre différents types d'orthèses. Un des axes de recherche à poursuivre en priorité est d'analyser l'impact de l'architecture (ou du principe) de l'orthèse sur son efficacité, l'observance et les effets secondaires [4]. Trois types génériques d'orthèses, chacune avec sa propre cinématique, permettent d'obtenir une avancée mandibulaire. Les mono-blocs, les bi-blocs en compression (ou en poussée) et les bi-blocs en retenue (ou en traction). Selon le consensus actuel, les orthèses sur mesure bi-blocs semblent mieux tolérées et diminuent le risque de complication au niveau des articulations temporo-mandibulaires [4].

L'étude que nous proposons vise donc à mettre en évidence le mécanisme d'action de ces deux grands types d'orthèse (bi-bloc en poussée - orthèse avec biellettes de Herbst et bi-bloc en retenue - orthèse de type "Optimisation de la Retenue Mandibulaire » ou O.R.M.) au moyen d'un modèle mécanique simple (voir figures 1 et 2). Ce modèle permet, objectif 
premier de l'étude, de comparer les ordres de grandeur des efforts musculaires et articulaires induits par ces orthèses.

\section{$\underline{\text { Matériels et méthodes }}$}

La mâchoire inférieure (ou mandibule) est reliée à l'os temporal par deux articulations temporo-mandibulaires. Chaque branche de la mandibule est dotée d'un condyle qui s'articule avec la fosse mandibulaire et le tubercule articulaire, par l'intermédiaire d'un disque. Les mouvements de la mâchoire sont des mouvements d'abaissement - élévation (ouverture/fermeture de la bouche), de propulsion-rétropulsion (abaissement puis translation antéro-postérieure de la mandibule, et retour) et de diduction (déplacement vers le bas et la droite ou la gauche suivant le côté travaillant). Dans le mouvement de propulsion, le condyle mandibulaire se déplace progressivement vers le bas et l'avant pour venir se placer sous le tubercule articulaire de l'os temporal, sous l'action des muscles ptérygoïdiens latéraux. La translation du condyle est essentiellement limitée par le frein temporo-méniscal et les ligaments stylo-mandibulaire et latéral [5].

Le modèle mécanique proposé est constitué de la mandibule, supposée rigide, en position de protrusion maximale (avancée de la mâchoire de $10 \mathrm{~mm}$ ). Le mécanisme considéré est symétrique par rapport au plan sagittal (d'un point de vue géométrique et de répartition des masses, mais également en ce qui concerne la transmission des efforts : muscles et ligaments). L'articulation entre le condyle et la zone temporale est modélisée par un contact ponctuel parfait (sans frottement). La force exercée par la fosse sur le condyle a alors une direction connue, perpendiculaire au profil de la fosse mandibulaire en position avancée. Celui-ci est représenté par un plan incliné de $35^{\circ}$ par rapport à l'horizontale [6]. Le centre du condyle, $\mathrm{O}$, est choisi comme origine du repère fixe. L'axe X est supposé parallèle au plan d'occlusion dans le plan de symétrie. La pesanteur s'exerce suivant l'axe -X sur un sujet couché, la masse et le centre de masse de la mandibule sont estimés d'après l'étude de Peck [5]. L'avancée de la mâchoire est obtenue par le biais d'une orthèse de type Herbst ou de type O.R.M. Le rôle des éléments freinateurs (i.e. essentiellement les ligaments) de la translation du condyle est modélisé par un élément élastique $\left(\mathrm{F}_{1}\right)$.

Figure 1

Figure 2

L'orthèse de Herbst est, depuis les années 80, l'orthèse d'avancée mandibulaire la plus utilisée en pratique clinique et la plus référencée dans les publications scientifiques sur le traitement du syndrome d'apnée obstructive du sommeil. Elle est constituée de deux gouttières qui se fixent sur les arcades dentaires inférieure et supérieure et reliées entre elles par deux biellettes métalliques dites de Herbst qui poussent la mandibule vers l'avant. L' orthèse de Herbst est représentée par une biellette inclinée, articulée par des liaisons pivots parfaites sur la mandibule d'une part et sur la mâchoire supérieure d'autre part (figure 1). L'architecture O.R.M. a été développée depu is 2002 par une équipe pluridisciplinaire dans le but d'offrir une alternative mieux tolérée par les patients. L'innovation principale réside dans l'articulation constituée d'une biellette et d'un triangle qui permet une cinématique d'avancée mandibulaire plus physiologique. Deux articulations relient les gouttières et retiennent la mandibule en position avancée. Les premiers résultats cliniques [7] semblent montrer une observance du traitement supérieure à celle rapportée dans la littérature sur les orthèses de 
Herbst [8]. L'orthèse O.R.M. est modélisée par une biellette, articulée par des liaisons pivots parfaites sur une pièce triangulaire solidaire de la mandibule d'une part et sur la mâchoire supérieure d'autre part (figure 2). Les muscles suivants sont pris en compte dans le modèle : masseter (m.), medial pterygoïd (m.p.), lateral pterygoïd (l.p.), anterior temporal (a.t.), posterior temporal (p.t.) et opener ou digastrique (op.). Leurs origines et insertions sont localisées dans le plan sagittal d'après l'article de Pruim [9]. Les sections physiologiques sont tirées de l'étude de Peck [5]. Ces données sont regroupées dans le tableau 1.

\section{Tableau 1}

L'équilibre statique de la mandibule conduit à un système d'équations hyperstatique, i.e. contenant plus d'inconnues ( 6 amplitudes de forces musculaires, l'amplitude de la réaction articulaire et de la force dans la biellette) que d'équations ( 3 équations d'équilibre dans le plan sagittal). La résolution de ce système passe par une optimisation numérique, consistant à minimiser un critère donné. Plusieurs critères de minimisation, proposés dans la littérature, sont testés : la somme des forces musculaires, la somme des contraintes musculaires au carré, la force articulaire [10], [6], [11]. Le choix de ces critères ici est avant tout physiologique, puisque pendant le sommeil, les muscles sont au repos et, idéalement, les articulations ne devraient pas être trop sollicitées.

L'équilibre mécanique des moments autour du centre du condyle doit être respecté, d'autre part les forces musculaires doivent rester inférieures à leurs valeurs maximales, données dans la littérature [9].

Le problème devient donc :

minimiser un critère : $\Sigma \mathrm{F}_{\mathrm{mi}}$, ou $\Sigma\left(\mathrm{F}_{\mathrm{m} /} / \mathrm{A}_{\mathrm{i}}\right)^{2}$ ou $\mathrm{R}_{\mathrm{o}}$

en respectant les contraintes suivantes : $\quad \overrightarrow{\mathbf{O C}} \wedge \overrightarrow{\mathbf{R}}+\sum_{i} \overrightarrow{\mathbf{O I}_{i}} \wedge \overrightarrow{\mathbf{F}_{m i}}=\overrightarrow{0}$

$0 \leq \mathbf{F}_{m i} \leq \mathbf{F}_{M A X, i}$

Où $R_{0}$ représente l'amplitude de l'action de contact articulaire, $C$ représente le point d'attache de la biellette sur la mandibule, $\mathrm{R}$ la force transmise dans la biellette. $\mathrm{I}_{\mathrm{i}}$ est le point origine $\mathrm{du}$ muscle $\mathrm{i}, \mathrm{F}_{\mathrm{mi}}$ la force développée par ce muscle, $\mathrm{A}_{\mathrm{i}}$ sa section physiologique maximale et $\mathrm{F}_{\mathrm{MAX}, \mathrm{i}}$ la force maximale qu'il est capable de dév elopper.

La résolution de ce problème est réalisée par optimisation numérique sous contraintes, en utilisant la fonction fmincon de Matlab.

\section{$\underline{\text { Résultats et Disc ussion }}$}

Les tableaux 2 et 3 donnent les valeurs des forces calculées respectivement pour les orthèses de type Herbst et O.R.M., en minimisant trois critères différents : la somme des forces musculaires $\left(\Sigma \mathrm{F}_{\mathrm{mi}}\right)$, la somme des contraintes musculaires au carré $\left(\Sigma\left(\mathrm{F}_{\mathrm{mi}} / \mathrm{A}_{\mathrm{i}}\right)^{2}\right)$ et l'amplitude de l'action de contact articulaire $\left(\mathrm{R}_{\mathrm{o}}\right)$. 
Tableau 2

Tableau 3

On constate que le critère utilisé a une influence sur les forces calculées. En ce qui concerne l'orthèse de type Herbst, tous les critères conduisent à une force importante dans les muscles releveurs de la mâchoire (masseter : de 12,6 à 16,2 $\mathrm{N}$ et posterior temporal : 69,9 N). Ces résultats confirment que cette orthèse a tendance à provoquer une ouverture de la bouche lorsque les muscles sont au repos. Au contraire, l'orthèse de type O.R.M. permet une protrusion de $10 \mathrm{~mm}$ avec un effort minime dans ces muscles (masseter : de 0,9 à $1,1 \mathrm{~N}$ et posterior temporal: 1,7 N). L'action de contact articulaire est également légèrement plus faible, de l'ordre de 10\%, avec l'orthèse de type O.R.M., ce qui pourrait se traduire par moins de phénomènes douloureux et expliquer la meilleure observance constatée en pratique clinique avec ce type d'orthèse. Cette concordance entre les résultats du modèle mécanique et les données cliniques est un élément de validation de notre démarche.

Les forces musculaires et articulaires calculées par notre modèle sont du même ordre de grandeur que celles trouvées par d'autres auteurs. Néanmoins, la plupart des études ont cherché à évaluer la force occlusive maximale pouvant être développée [12], [13], [14]. A titre d'exemple, le modèle tridimensionnel développé par Peck [5] pour simuler l'ouverture maximale de la bouche, qui correspond à la condition de fonctionnement la plus proche de celle que nous avons considérée, conduit à des forces de $28 \mathrm{~N}$ sur l'articulation et respectivement $16,8 \mathrm{~N}$ pour le lateral pterygoïd, $9 \mathrm{~N}$ pour le masseter et $4,6 \mathrm{~N}$ pour le posterior temporal.

Si les données expérimentales ou les modélisations de conditions similaires manquent dans la littérature pour valider plus avant notre modèle, nous pouvons toutefois affirmer que la comparaison des deux orthèses, avec des caractéristiques de la mandibule et musculaires identiques, est fiable.

Il est à noter que les résultats présentés ici correspondent à des paramètres standard de design des orthèses d'avancée mandibulaire (longueurs et inclinaisons des biellettes). La variation de ces paramètres modifie légèrement les résultats sans toutefo is changer les ordres de grandeur et la tendance observée.

\section{Conclusion - Perspectives}

Ce modèle mécanique simple a permis de comparer deux types d'orthèses d'avancée mandibulaire utilisées dans le traitement des syndromes d'apnées obstructives du sommeil. Cette comparaison a été réalisée en termes d'efforts musculaires et d'action de contact induite sur l'articulation. Les efforts calculés sont en accord avec les ordres de grandeurs trouvés dans la littérature, utilisant des modélisations similaires pour des applications différentes.

Les résultats de ce modèle montrent principalement que l'orthèse de type Herbst induit nécessairement une ouverture de la bouche lorsque les muscles sont au repos, ce qui n'est pas le cas avec l'orthèse de type O.R.M. Il ressort également une légère diminution de l'action de contact avec l'orthèse de type O.R.M. Ces résultats tendent à montrer un meilleur profil clinique en termes de compliance et d'effets secondaires des orthèses travaillant en retenue par rapport à celles travaillant en compression. En effet, un consensus médical établit que la 
respiration physiologique durant le sommeil devrait s'effectuer par le nez, bouche fermée et que les forces de contact subies par l'articulation temporo-mandibulaire doivent être minimisées pour réduire l'inconfort et les effets secondaires des orthèses.

Pour aller plus loin dans l'analyse, en particulier mieux caractériser l'impact sur les dents (par l'intermédiaire des gouttières) et envisager une optimisation de l'architecture de ces orthèses, une modélisation plus fine, de type éléments finis, est envisagée.

\section{$\underline{\text { Références }}$}

[1] Young T, Peppard PE, Gottlieb DJ. Epidemiology of obstructive sleep apnea: a population health perspective. Am J Respir Crit Care Med 2002;165:1217-39.

[2] Clark GT, Blumenfeld I, Yoffe N, Peled E, Lavie P. A crossover study comparing the efficacy of continuous positive airway pressure with anterior mandibular positioning devices on patients with obstructive sleep apnea. Chest 1996;109:1477-83.

[3] Pantin CC, Hillman DR, Tennant M. Dental side effects of an oral device to treat snoring and obstructive sleep apnea. Sleep 1999;22:237-40.

[4] Cistulli PA, Gotsopoulos H, Marklund M, Lowe AA. Treatment of snoring and obstructive sleep apnea with mandibular repositioning appliances. Sleep Med Rev $2004 ; 8: 443-57$.

[5] Peck CC, Langenbach GE, Hannam AG. Dynamic simulation of muscle and articular properties during human wide jaw opening. Arch Oral Biol 2000;45:963-82.

[6] Nickel JC, Iwasaki LR, Walker RD, McLachlan KR, McCall WD, Jr. Human masticatory muscle forces during static biting. J Dent Res 2003;82:212-7.

[7] Navailles B, Pignat JC. Efficacy and compliance of novel mandibular advancement device. 6th IFOS. Roma, 2005.

[8] Mc Gown AD, Spiro SF. Long term use of mandibular advancement splint for snoring and OSA: a questionnaire survey. European Respiratory Journal 2001;17:462-466.

[9] Pruim GJ, de Jongh HJ, ten Bosch JJ. Forces acting on the mandible during bilateral static bite at different bite force levels. J Biomech 1980;13:755-63.

[10] Koolstra JH, van Eijden TM. A method to predict muscle control in the kinematically and mechanically indeterminate human masticatory system. J Biomech 2001;34:117988.

[11] Iwasaki LR, Baird BW, McCall WD, Jr., Nickel JC. Muscle and temporomandibular joint forces associated with chincup loading predicted by numerical modeling. Am J Orthod Dentofacial Orthop 2003;124:530-40.

[12] Osborn JW, Baragar FA. Pred icted pattern of human muscle activity during clenching derived from a computer assisted model: symmetric vertical bite forces. J Biomech $1985 ; 18: 599-612$.

[13] Faulkner MG, Hatcher DC, Hay A. A three-dimensional investigation of temporomandibular joint loading. J Biomech 1987;20:997-1002.

[14] Trainor PG, McLachlan KR, McCall WD. Modelling of forces in the human masticatory system with optimization of the angulation s of the jo int loads. J Biomech $1995 ; 28: 829-43$. 
Tableau 1 : Géométrie, sections physiologiques et forces maximales musculaires.

\begin{tabular}{|c|c|c|c|c|}
\hline & $\begin{array}{l}\text { Bras de levier / origine } \\
\text { (cm) }\end{array}$ & $\begin{array}{c}\text { Inc lina is on / } \\
\text { horizonta le X }\left(^{\circ}\right)\end{array}$ & $\begin{array}{l}\text { Section physiologique } \\
\left(\mathrm{cm}^{2}\right)\end{array}$ & Force maximale $(\mathrm{N})$ \\
\hline Masseter & 4,1 & 64,1 & 6,8 & 272 \\
\hline $\begin{array}{l}\text { medial } \\
\text { pterygoïd }\end{array}$ & 3,5 & 70,3 & 4,37 & 174,8 \\
\hline $\begin{array}{l}\text { lateral } \\
\text { pterygoïd }\end{array}$ & 0 & 0 & 1,67 & 66,9 \\
\hline $\begin{array}{l}\text { anterior } \\
\text { temporal }\end{array}$ & 3,3 & 99,5 & 3,95 & 158 \\
\hline $\begin{array}{l}\text { posterior } \\
\text { temporal }\end{array}$ & 2,1 & 139,8 & 1,89 & 75,6 \\
\hline $\begin{array}{l}\text { anterior } \\
\text { digastrique }\end{array}$ & $-10,4$ & 210,8 & 1 & 40 \\
\hline
\end{tabular}

Tableau 2: Forces calculées par les différents critères pour une orthèse de type Herbst

\begin{tabular}{|c|c|c|c|}
\hline Force & $\Sigma \mathrm{F}_{\mathrm{mi}}$ & $\sum\left(\mathrm{F}_{\mathrm{mi}} / \mathrm{A}_{\mathrm{i}}\right)^{2}$ & $\mathrm{R}_{\mathrm{o}}$ \\
\hline Biellette & $48 \mathrm{~N}$ & $35,9 \mathrm{~N}$ & $113,8 \mathrm{~N}$ \\
\hline Contact & $43,1 \mathrm{~N}$ & $47,9 \mathrm{~N}$ & 0 \\
\hline Masseter & $16,2 \mathrm{~N}$ & $12,6 \mathrm{~N}$ & $69,9 \mathrm{~N}$ \\
\hline Post. temporal & 0 & 0 & 0 \\
\hline Lat. pterygoïd & 0 & $15,9 \mathrm{~N}$ & 0 \\
\hline
\end{tabular}


Tableau 3 : Forces calculées par les différents critères pour une orthèse de type ORM

\begin{tabular}{|c|c|c|c|}
\hline Force & $\Sigma \mathrm{F}_{\mathrm{mi}}$ & $\Sigma\left(\mathrm{F}_{\mathrm{m}} / \mathrm{A}_{\mathrm{i}}\right)^{2}$ & $\mathrm{R}_{\mathrm{o}}$ \\
\hline Biellette & $48,6 \mathrm{~N}$ & $38,9 \mathrm{~N}$ & $50,1 \mathrm{~N}$ \\
\hline Contact & $39 \mathrm{~N}$ & $43,7 \mathrm{~N}$ & $36,9 \mathrm{~N}$ \\
\hline Masseter & $0,9 \mathrm{~N}$ & $1,1 \mathrm{~N}$ & 0 \\
\hline Post. temporal & 0 & 0 & $1,7 \mathrm{~N}$ \\
\hline Lat. pterygoïd & 0 & $13,1 \mathrm{~N}$ & 0 \\
\hline
\end{tabular}

Figure 1:

a. Photo d'une orthèse de type Herbst

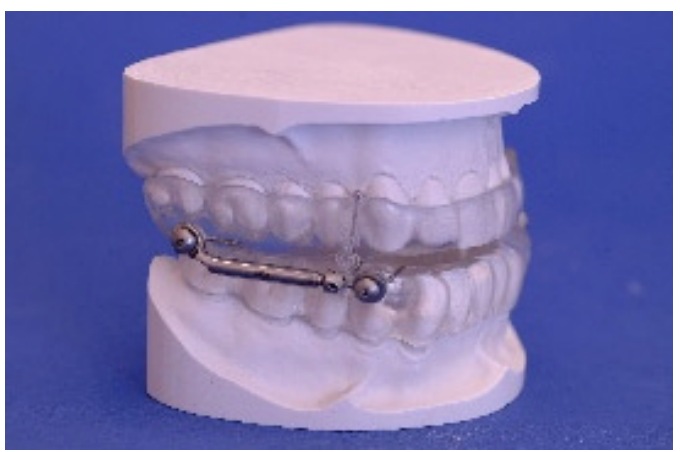

b. Schéma avec orthèse de type Herbst en place (patient couché) 


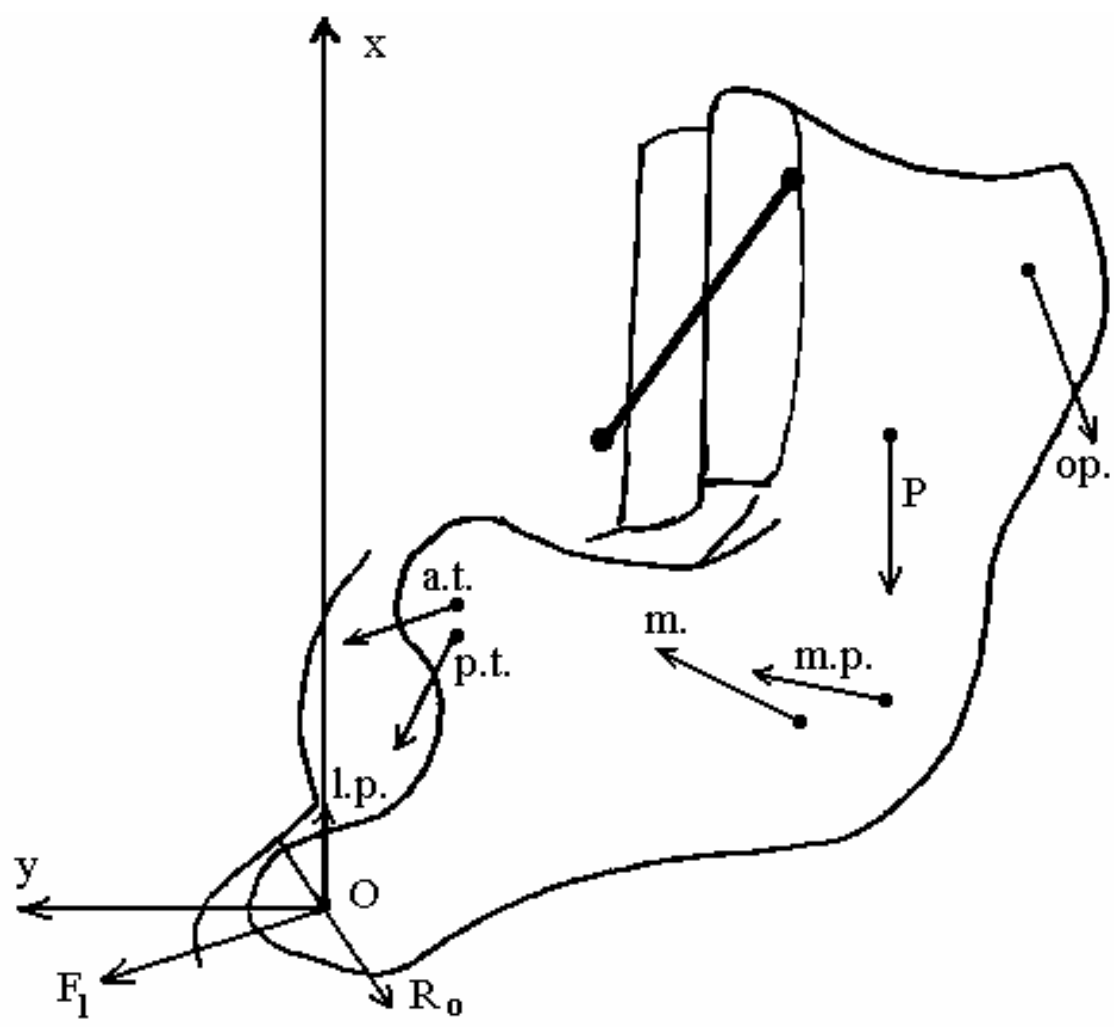

Figure 2 :

a. Photo d'une orthèse de type ORM

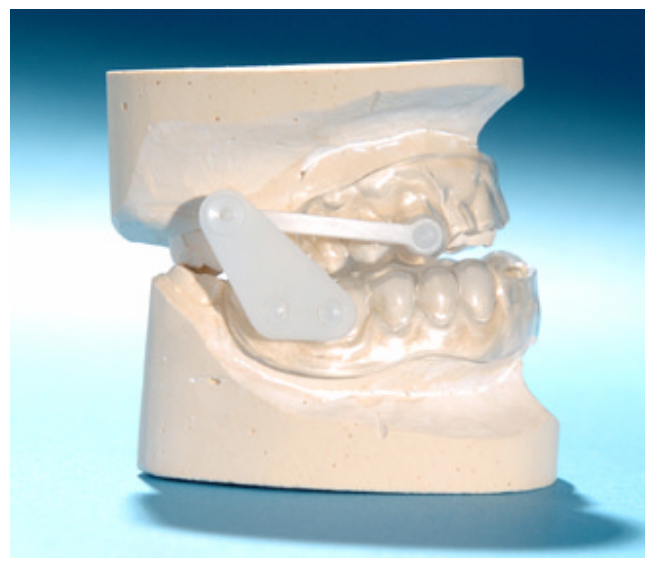

b. Schéma avec orthèse de type ORM en place (patient couché) 


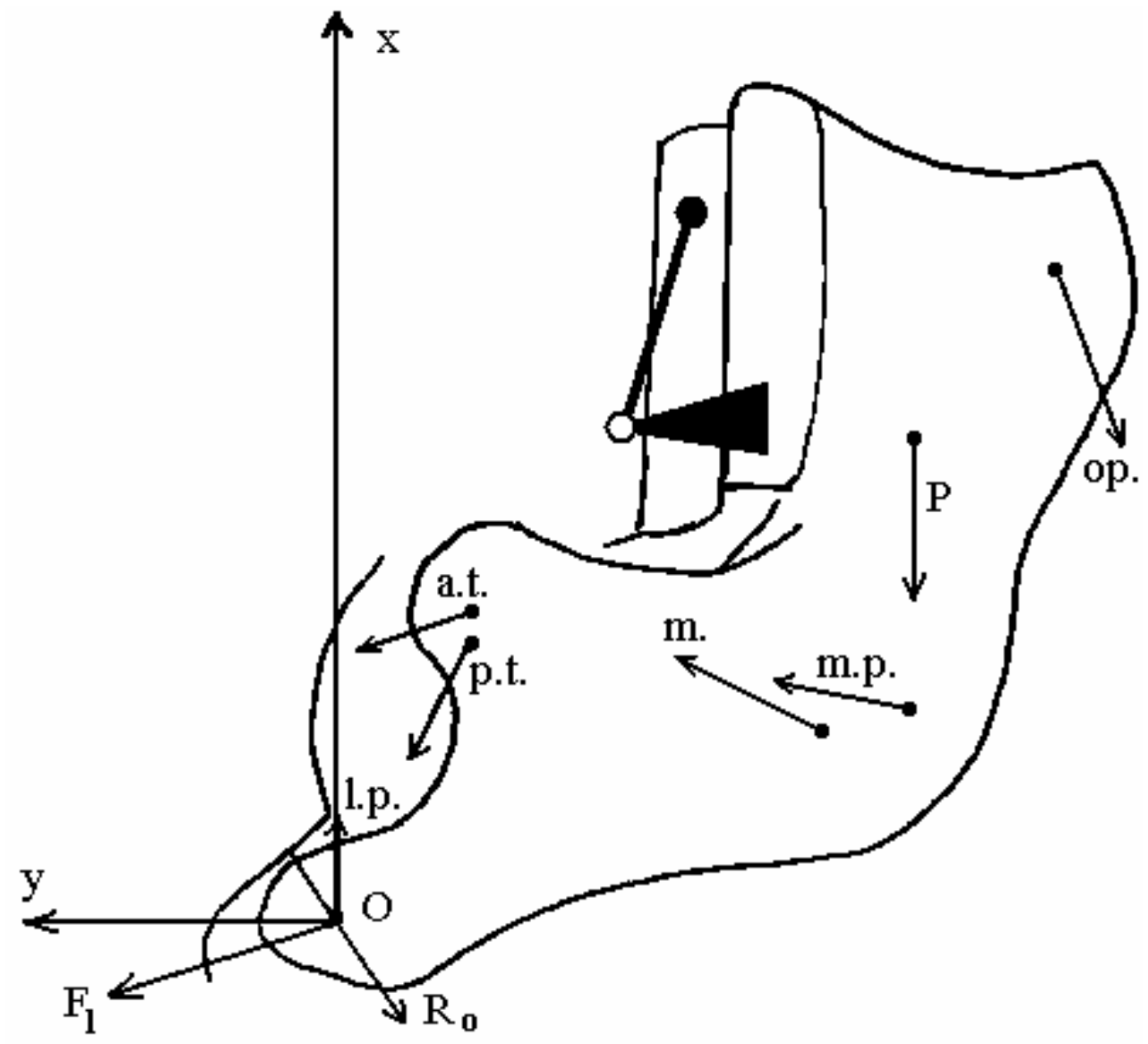


Ms. Ref. No.: RBMRET-D-07-00001

Title: Impact des orthèses d'avancée mand ibulaire sur l'articulation temporo-mand ibulaire ITBM-RBM, Innovation et Technologie en Biologie et Médecine

\section{Réponse point par point au reviewer \#1}

Les auteurs remercient le reviewer pour ses nombreuses remarques très justes et constructives. Les corrections suggérées ont été prises en compte, comme détaillé ci-dessous :

Titre, ligne 1 : Impact d'orthèses ... Seules deux architectures sont évaluées ici. Effectivement, le titre a été corrigé en "Impact de deux orthèses ... »

Les architectures assurant traction ou retenue doivent être plus lisibles et définies. Deux photos explicites peuvent enrichir les figures 1 et 2 (site Sté Narval, par exemple) et compléter le texte en donnant lieu à un renvoi (lignes 12, 110 et 121). Ceci permettra de souligner l'objectif énoncé (ligne 75).

Des photos permettant de mieux visualiser les deux types d'orthèses étudiées ont été ajoutées, figure 1. a. et 2. a. avec renvois dans le texte.

L'expression sys tème d'équations redondant (ligne 13) doit être explicitée (une définition de système d'équations hyperstatique est donnée ligne 130). Le terme redondant n'est-il pas, par ailleurs, ambigu et à remplacer ? Pour ce modèle plan, les inconnues scalaires sont au nombre de 8 si toutes les directions sont connues ( 6 paires de muscles, 1 paire de biellettes et 1 contact articulaire ponctuel dans le plan sagittal).

Le terme "redondant», plus utilisé dans le domaine de la robotique, a été remplacé par " hyperstatique ». La définition du système hypersta tique a également été davantage explicité : "L'équilibre statique de la mandibule conduit à un système d'équations hyperstatique, i.e. contenant plus d'inconnues (6 amplitudes de forces musculaires, l'amplitude de la réaction articulaire et de la force dans la biellette) que d'équations (3 équations d'équilibre dans le plan sagittal). »

Les retouches proposées ont été également faites.

$>$ Ligne 2 : code postal 69000 et Université $\Rightarrow O K$

$>$ Ligne 12 : Inverser les termes traction et poussée. L'orthèse en poussée précède ensuite l'orthèse en traction dans la présentation. Faut-il privilégier le terme traction ou le terme retenue (rétention) utilisé plus loin ? Retenue apparaît dans le sigle ORM de la Sté Narval. $\Rightarrow$ Le terme " retenue » a été choisi et systématiquement utilisé à la place de " traction"

$>$ Ligne $26: \ldots$ des orthèses $\ldots \Rightarrow O K$

$>$ Ligne 32 : Remplacer, "Understand mechanical forces applied on the temporo-mandibular joint by two different designs of mandibular advancement devices." $\Rightarrow O K$

$>$ Lignes 33 et $34: \ldots$ temporo-mandibular joint. $\Rightarrow O K$

$>$ Ligne 35: Phrase à revoir "A comparative study of traction based vs..." $\Rightarrow$ «A study is designed to compare traction based vs. compression based devices, with mandible in a $10 \mathrm{~mm}$ protrusion position."

$>$ Ligne 37 et 38 : Vérifier que l'anglais n'écrive pas hyper static en deux mots ? $\Rightarrow$ En anglais, on trouve plus couramment « hyperstatic » et « hyper-static»

$>$ Ligne $46:$ Couper la phrase $\ldots$ of action. The results $\ldots \Rightarrow O K$

$>$ Ligne $48: \ldots$ an improved side-effect and a compliance profile... $\Rightarrow O K$

$>$ Ligne 51: Majuscules aux mots-clés $\Rightarrow O K$

$>$ Ligne $54:$....adulte [1] et dont les ... $\Rightarrow O K$

$>$ Lignes 56 et $57:$ Revoir la rédaction, "Le traitement de référence est la ventilation par pression positive continue (VPPC) qui est une attelle pneumatique prévenant le collapsus pharyngé". $\Rightarrow$ "Le traitement de référence est la ven tilation par pression positive continue (VPPC), obtenue par une attelle pneumatique ... » 
$>$ Ligne $68: . .$. d'orthèses. $\Rightarrow O K$

$>$ Lignes 68 et 69 : Le terme design n'a-t-il pas un sens voisin d'architecture ici ?

"Un des axes de recherche à poursuivre en priorité est d'analyser l'impact de l'architecture (ou du principe) de l'orthèse sur son efficacité." $\Rightarrow O K$

$>$ Ligne $70:$ Trois types génériques d'orthèses, chacune avec $\ldots \Rightarrow O K$

$>$ Ligne 77 : In troduire la définition "optimisation de la retenue mandibulaire" avant d'utiliser la désignation O.R.M. et, par suite, supprimer cette expression à la ligne 115 (il s'agit d'ailleurs plus d'une architecture de conception à conséquen ces mécaniques que d'une technologie). $\Rightarrow$ correction Ligne 77 puis remplacement de " technologie » par " architecture ORM»

$>$ Ligne 77 : Remplacer la seconde phrase du paragraphe par "Ce modèle permet, objectif premier de l'étude, de comparer les ordres de grandeur des efforts musculaires et articulaires induits par ces orthèses." Le modèle d'étude doit être considéré comme l'outil (titre). Eviter la répétition des lignes 68 et $69 . \Rightarrow O K$

$>$ Ligne 90 : La capsule articulaire est en fait "relâchée" à l'arrière du condyle en position repos. L'amplitude du déplacement est très probablement essentiellement limitée par les ligaments. $\Rightarrow$ La phrase a été remplacée par : "La translation du condyle est essentiellement limitée par le frein temporo-méniscal et les ligaments stylo-mandibulaire et latéral »

> Ligne 96 : Remplacer ...son logement (fosse mandibulaire)... par, la zone temporale. Même remarque lignes 97 et 98 . Sous protrusion de $10 \mathrm{~mm}$, la situation du "point" de contact condyle/zone temporale résulte de la géométrie articulaire (pente, type facial...) mais se situe au voisinage du condyle temporal. Confirmation en ligne $99 . \Rightarrow O K$

$>$ Ligne 104 : Expliciter éléments freinateurs. $\Rightarrow$ Le rôle des éléments freinateurs (i.e. essentiellement les ligaments) de la translation du condyle...

$>$ Ligne 140: Justifier le choix des trois critères proposés (points de vue physio. ou méca.). Minimiser R0 a une incidence directe sur l'état de sollicitation du ménisque lors du repos du patient. Introduire la remarque des lignes 198 et 199 à ce stade, dans le choix donc du mode de résolution du système d'équations. $\Rightarrow$ Une phrase a été ajou tée pour justifier le choix de ces critères : "Le choix de ces critères ici est avant tout physiologique, puisque pendant le sommeil, les muscles sont au repos et, idéalement, les articulations ne devraient pas être trop sollicitées».

Ligne 165 : Rapprocher le constat "L'action de contact articulaire ... ce type d'orthèse." du choix des critères, ligne 140 . Revenir, donc, à l'hypothèse après la résolution pour valider la démarche. $\Rightarrow$ Une phrase a été ajoutée dans ce sens: "Cette concordance entre les résulta ts du modèle mécanique et les données cliniques est un élément de validation de notre démarche». 


\section{Figure}

Click here to downldad high resolution image

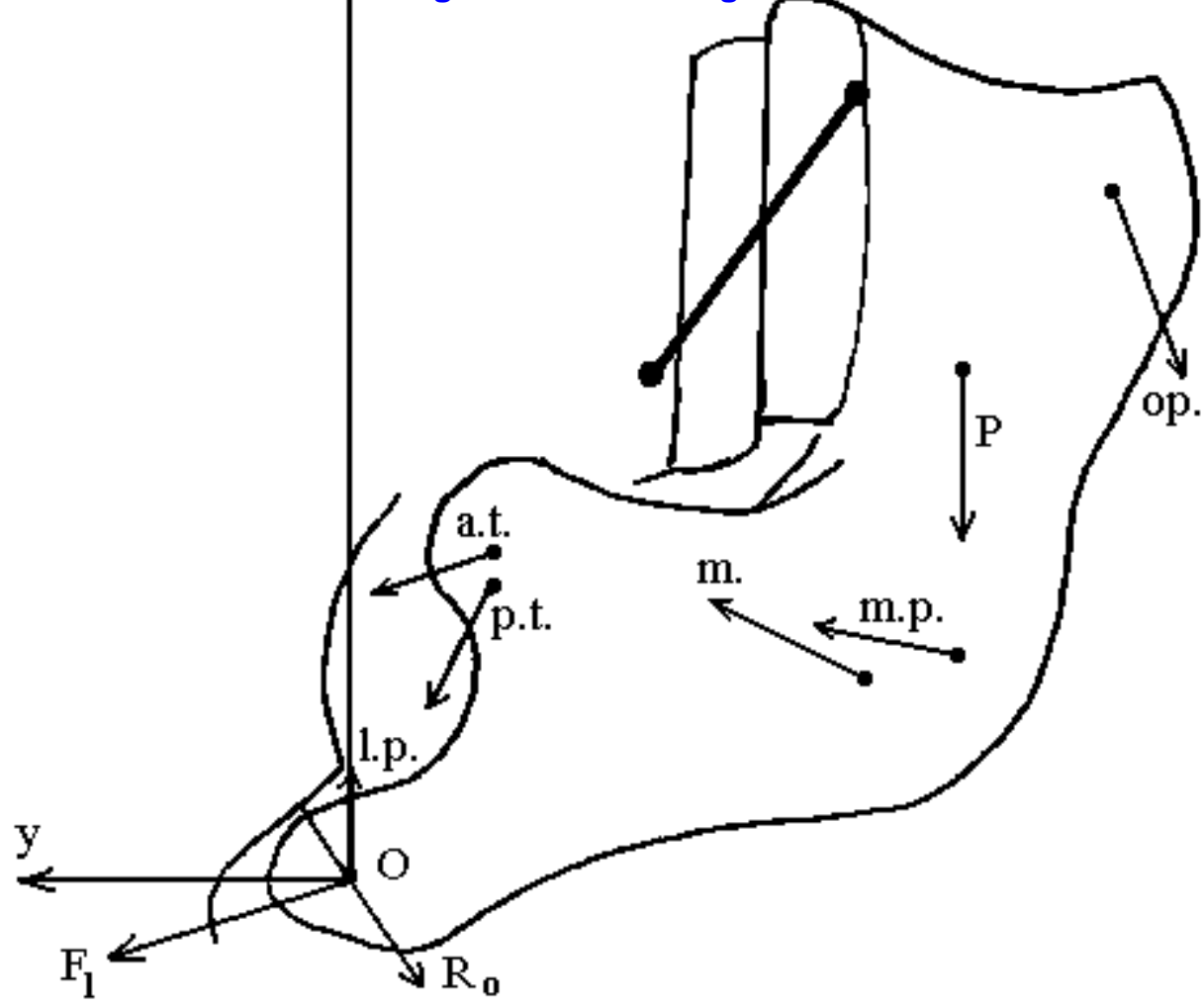



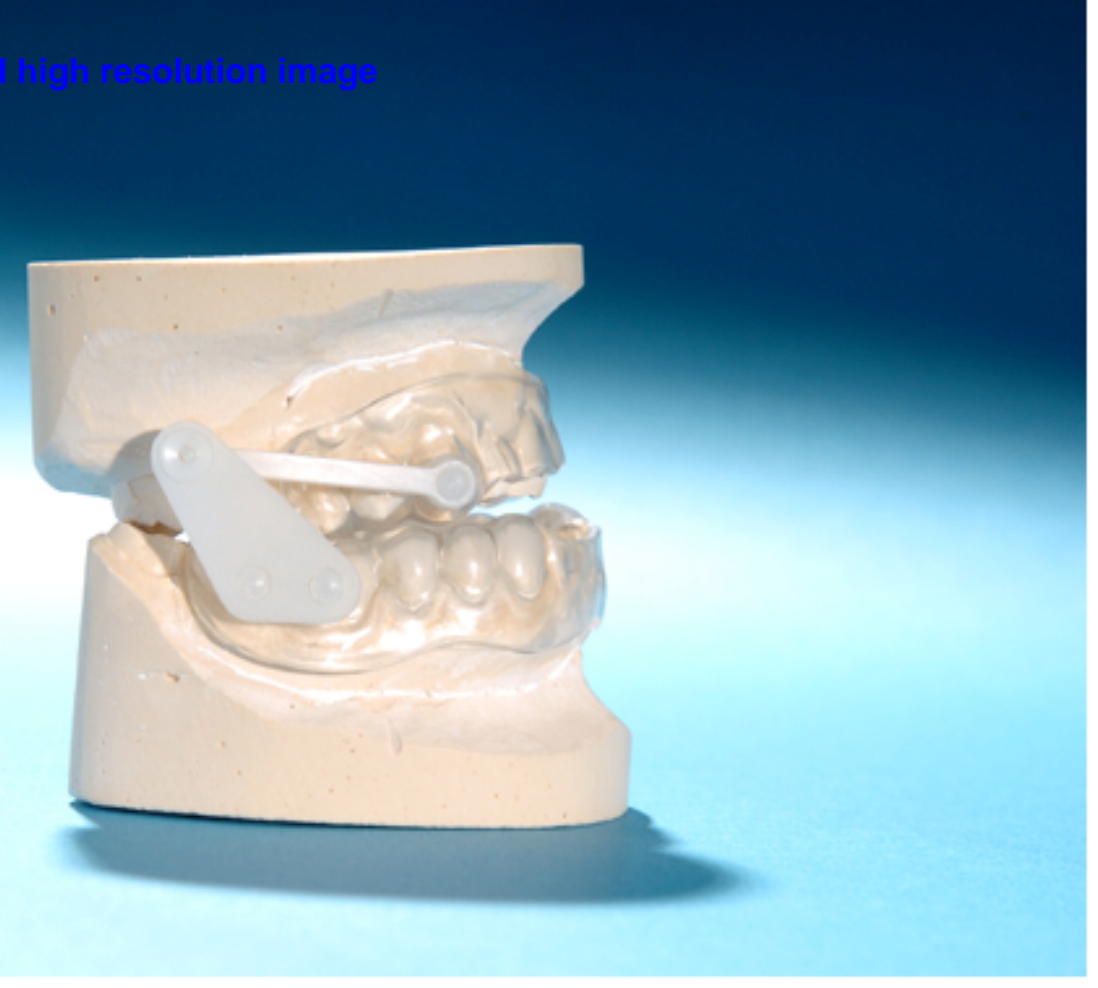


\section{Figure}

Click here to downldad high resolution image

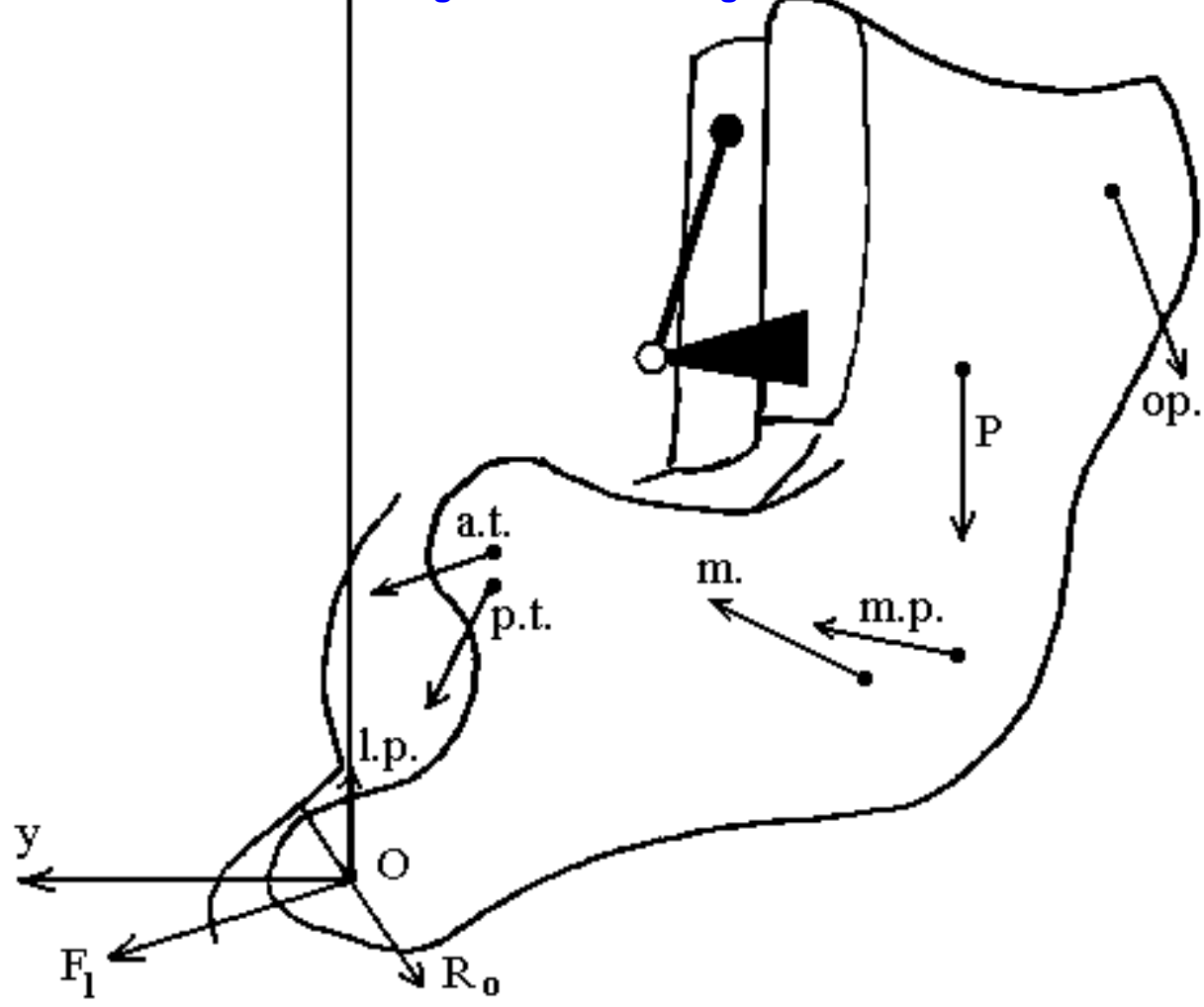

